\title{
Development of a shutterless calibration process for microbolometer-based infrared measurement systems
}

\author{
by A. Tempelhahn*, H. Budzier*, V. Krause* and G. Gerlach*
}

\author{
* Solid-State Electronics Laboratory, Technische Universität Dresden, 01062 Dresden, Germany, \\ alexander.tempelhahn@tu-dresden.de
}

\begin{abstract}
To measure temperatures by microbolometer-based infrared cameras one has to deal with disturbing radiation flux due to the ambient temperature derived from the interior of the camera enclosure. The standard approach determines this radiation flux by using an optical shutter occasionally and subtracting the derived correction signal from the measurement signal. Omitting mechanical parts like the optical shutter might lead to more constructive freedom which is needed for advanced miniaturization. For that reason, a different compensation method and thus calibration process has to be developed. This paper proposes novel approaches to overcome the influences of changing ambient temperatures and discusses the conditions aiming for a new method.
\end{abstract}

\section{Introduction}

Microbolometers belong to the class of thermal infrared sensors [1]. The working principle is based on the temperature dependence of the electric resistance of the sensor material. The absorbed incident radiation changes the temperature of the bolometer elements and, hence, the electric resistance changes. A readout integrated circuit (ROIC) converts the resistance change into a signal voltage proportional to the exchanged radiant flux. Additional knowledge of the radiation source properties and the transmission path allows determining the temperature of the radiator.

There are other types of thermal infrared sensors like thermopiles and pyroelectric detectors [1]. However, working uncooled is the most important advantage of thermal infrared sensors in comparison to photon sensors because it enables cost reduction of IR camera systems. This resulted in the widespread usage of thermal infrared sensors, especially microbolometer focal plane arrays (FPA), in the field of low-cost thermographic applications.

Advanced packaging technologies lead also to smaller and smaller infrared FPAs. Sensor manufacturers make a lot of efforts to combine all necessary electrical environments into one single chip (system on chip - SoC); including analog BIAS voltages, readout integrated circuits (ROIC) und analog-to-digital converters (ADC) [2]. Such all-in-one infrared sensors will be suitable for the growing field of applications in building security, automotive devices or smart phone devices. With respect to the boundary conditions in these application fields, those systems should be small, simple-constructed and durable. Present devices of this kind are mostly limited to infrared imaging applications and are not as precise as present shutter-based radiometrically calibrated infrared measurement systems.

Microbolometers are sensitive over a broad spectral bandwidth. The sensitivity is defined by construction parameters like the width of the $\alpha / 4$-absorber and the used filter window covering the FPA. In most cases they are optimized for high sensitivity in the long wavelength infrared spectral band. Disturbing radiation derived from the interior of the camera is the main source of measurement uncertainty and has to be considered for quantitative measuring applications. The common approach is to determine the amount of disturbing radiation by using an optical shutter. Though, mechanical parts limit the size of the devices and are vulnerable to shocks and temperature changes, omitting them is desirable.

\section{Radiation model and thermal drift}

Each object with a temperature above absolute zero emits radiation corresponding to the Planck's Law (Equ. 1). As mentioned above, this is used in thermal infrared sensors for non-contact temperature measuring. Beside the object temperature also material properties, like transparency, and surface properties, like structure texture, have a huge influence on the amount of emitted radiant flux. Adjustable radiation sources with high and uniform radiance over a discrete surface area are called blackbodies. These radiators are essential during the calibration process of infrared measurement systems. The ratio of effectively emitted and the maximum possible spectral radiance from an ideal blackbody is called emissivity $\varepsilon$. Real radiators never reach an emissivity of unity. Figure 1 shows the spectral radiance of an ideal blackbody at different temperatures described by the Planck's Law.

$$
M_{\lambda S}=\frac{c_{1}}{\lambda^{5}} \frac{1}{e^{\frac{c_{2}}{\lambda T}}-1}
$$


where $M_{\lambda S}$ is spectral radiance, $\lambda$ spectral wavelength, $T$ object temperature and $c_{1}=3.741775 \mathrm{E} 16 \mathrm{~W} \mathrm{~m} \mathrm{~m}^{2}$ and $c_{2}=$ $1.438769 \cdot 10^{-2} \mathrm{~m} \mathrm{~K}$ are constants.

In most cases radiation passes the atmosphere from the emitting source to the observing infrared camera. The surrounding atmosphere consists of radiation-absorbing molecules, mainly oxygen, carbondioxide and water vapor, absorbing parts of the radiation. Hence, it is not possible to detect the entire radiation over the complete infrared spectral band. There are three atmospheric windows in the near $(1.2 \ldots 2.4 \mu \mathrm{m})$, middle $(3.2 \ldots 5.2 \mu \mathrm{m})$ and far $(8 \ldots 13 \mu \mathrm{m})$ infrared spectral band [1]. As mentioned above microbolometer sensors are particularly used for measurement application in the far infrared spectral band.

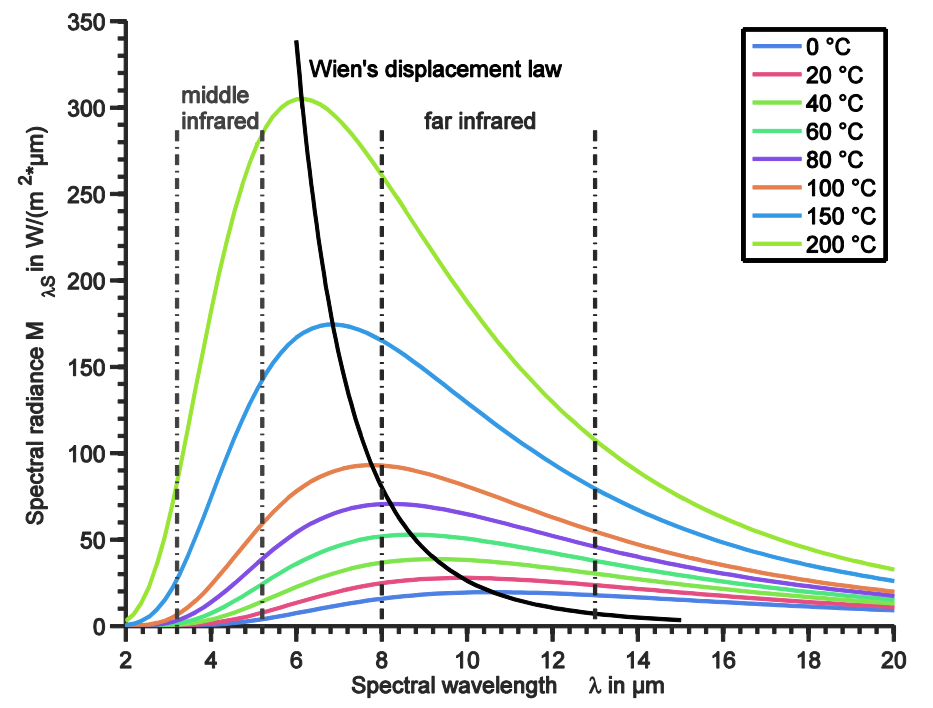

Fig. 1. Spectral radiance of an ideal blackbody at several temperatures.

The spectral wavelength at the maximum of spectral radiance depends on the object temperature and follows the Wien's displacement law (Fig. 1). Objects at ambient temperature including the housing of the infrared camera have a spectral peak wavelength of emission in the far infrared spectral band. Caused by the huge field of view (FOV) of the infrared sensor [3] it detects radiation from the observed scene as well as disturbing radiation derived from the interior of the camera housing. Figure 2 shows the main influences on the radiation measurement and illustrates the ambient temperature dependency. Using a lens system with an $f$-number of unity, which is often applied, leads to a resultant ratio of $F O V_{\text {scene }}$ to $F O V_{\text {sensor }}$ of about 0.2 [1]. This fact makes the temperature distribution inside the camera to a decisive source of measurement uncertainty.

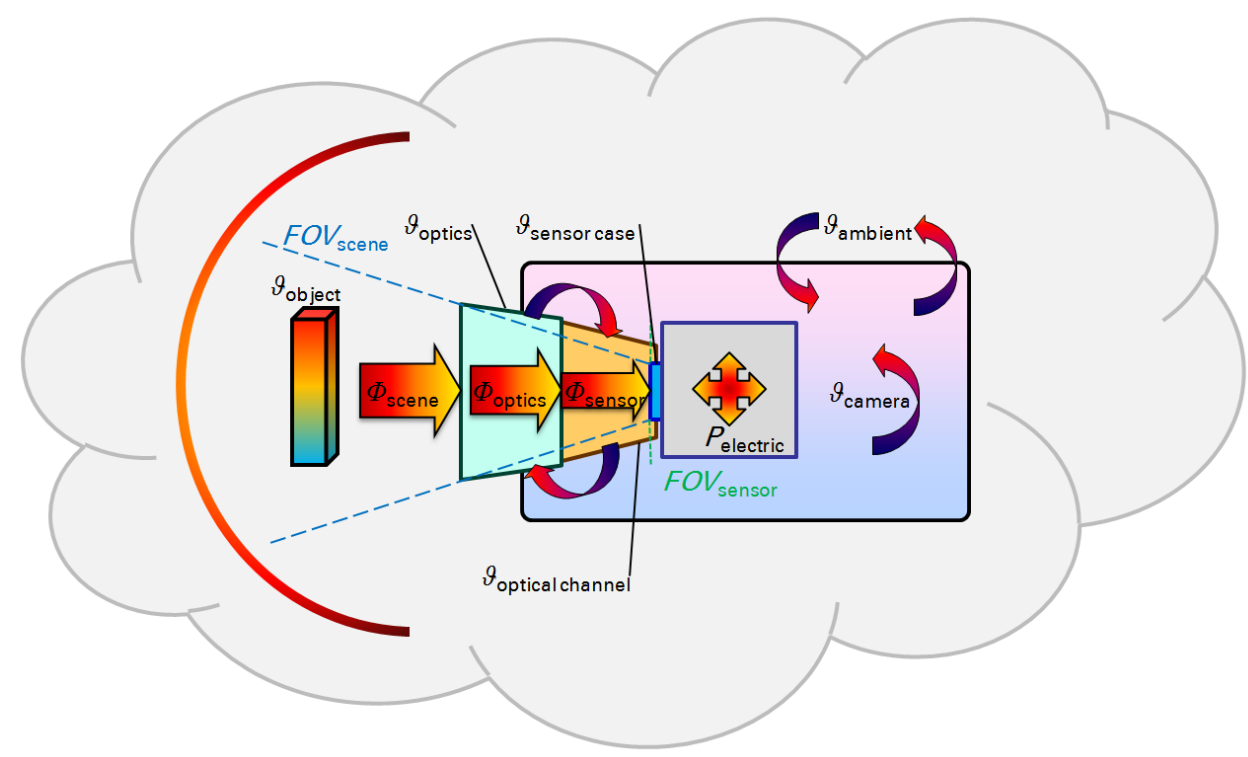

Fig. 2. Model of an infrared camera comprising sensor, optics, housing, electronics and measurement environment [3]. $\vartheta_{\text {object }}$ is the object temperature, $\vartheta_{\text {optics }}, \vartheta_{\text {sensor case }}, \vartheta_{\text {optical channel }}, \vartheta_{\text {camera }}, \vartheta_{\text {ambient }}$ are the temperature of the optics, the sensor case and so on. FOV $V_{\text {sensor }}$ illustrates the wide sensor field of view compared to the camera field of view FOV scene $_{\text {. }}$ electric mentions the thermal losses. 
Figure 2 shows a model of an IR camera which considers the camera with its components as part of the measurement set-up including the relevant temperatures. The exchanged radiation $E_{\text {sensor }}$ of the infrared sensor can be assumed as sum of the detected irradiance $E_{\text {scene }}$ from the observed scene and the camera interior $E_{\text {camera }}$ minus the radiant exitance $M_{\text {sensor }}$ of the sensor:

$$
E_{\text {sensor }}=E_{\text {scene }}+E_{\text {camera }}-M_{\text {sensor }}
$$

Since $E_{\text {scene }}$ depends on the temperature distribution of the object to be measured and the scene background, it is the interesting portion of radiation. The sensor array consists of numerous single infrared sensor elements placed in columns and rows. An infrared transmitting lens system images each part of the observed scene onto the single pixels. The aperture size determines the solid angle $\omega_{O b j}$ which is related to the mean surface temperature $\vartheta_{O b j}$ of the corresponding part of the object space. Because each pixel has its own specific position related to the aperture, the object solid angle $\omega_{o b j}$ varies from pixel to pixel [2].

The radiation derived from the camera interior meets the pixels unfocused. The corresponding solid angle $\omega_{\text {Cam }}$ is also pixel-dependent. The amount of disturbing radiant flux depends on the temperature distribution $\delta \vartheta_{\text {Cam }}$ inside the camera. It is influenced by several conditions, particularly the heat conduction of the ambient temperature and the conduction as well as the convection of heat generated inside the camera due to thermal losses of the digital signal-processing unit. Since the thermal losses can be assumed to be constant, only the ambient temperature is an independent variable. The main task in quantitative radiation measurements are the inhomogeneous temperature distribution and its non-uniform response on ambient temperature changes. Determining the amount of disturbing radiant flux is essential to reduce the measurement uncertainty. The less the effect of ambient temperature on measurement is corrected, the less the measurement result is reliable. As mentioned above the relation between temperature and radiant exitance is nonlinear. Therefore, the disturbing radiant flux should be measured directly using the infrared sensor itself.

The working temperature $\vartheta_{F P A}$ of the sensor can be assumed to be uniform over the complete FPA. It defines the responsivity $R_{V}$ and offset voltage $V_{0}$ of each pixel. Due to its huge influence on the radiation measurement, however, former microbolometer sensor arrays were temperature-stabilized by an integrated thermoelectric cooler (TEC) [1]. This stabilization is a power-demanding procedure. Nowadays, TEC-less FPAs are also available and used particularly for compact devices. Using the sensor temperature as parameter it is possible to correct the variation in responsivity and offset derived from the changing sensor temperature [4]. After this correction or using a temperature stabilized FPA the sensor parameter can be assumed to be constant. The output voltage of a sensor pixel can be derived from Equ. (2):

$$
V_{P i x}=R_{V} A_{P i x}\left[\omega_{O b j} L\left(\vartheta_{O b j}\right)+\int_{\omega_{C a m}} L\left(\delta \vartheta_{C a m}, \omega\right) d \omega-M_{P i x}\right]+V_{0}
$$

Here $V_{\text {Pix }}$ is the signal voltage, $R_{V}$ the responsivity, $A_{P i x}$ the pixel sensor area, $\omega_{\text {Obj }}$ the solid angle to object, $\vartheta_{\text {Obj }}$ the object temperature, $\omega_{\text {Cam }}$ the solid angle to camera, $\delta \vartheta_{\text {Cam }}$ the camera temperature distribution, $M_{\text {Pix }}$ pixel radiant exitance and $\mathrm{V}_{0}$ the offset voltage. It is assumed that the blackbody radiation covers the entire $F O V_{\text {scene }}$ and, hence, the object irradiance $L\left(\vartheta_{O b j}\right)$ is homogeneous over the complete object solid angle $\omega_{O b j}$.

\section{Pixel non-uniformity}

Microbolometer infrared sensor arrays are manufactured by microfabrication. Despite all improvements in fabrication precision small variations in process parameters lead to inhomogeneous pixel-specific sensor parameters responsivity $R_{V, i j}$ and offset voltage $V_{0, i j}$.

$$
V_{i j}=R_{V, i j} \Phi_{i j}+V_{0, i j}
$$

Therefore, even if the amount of exchanged radiant flux $\Phi_{i j}$ is the same for each pixel $(i, j)$, the response signal voltage $V_{i j}$ would vary in certain limits (Equ. 4). This pixel non-uniformity has to be corrected by digital signal-processing algorithms in order to receive pixel signal voltages responding in the same way to the amount of detected irradiance. This means that - if the infrared camera is directed to a homogeneous radiation source (e.g. a blackbody) which covers the entire camera field of view - the received infrared image should be homogeneous as well.

As Equ. (4) shows the signal voltage $V_{i j}$ is linearly dependent to the exchanged radiant flux $\Phi_{i j}$. The non-uniformity results in different values for gain $R_{V, i j}$ and offset $V_{0, i j}$ of each pixel's response curve. Figure 3 shows the principle of the linear non-uniformity correction (NUC) procedure for two pixels. The black curve illustrates the mean response curve of all pixels, called normal curve $\left\langle V_{i j}\right\rangle$. Because of the linear relation between radiation and signal voltage, a two-point correction method is sufficient [4]. Equ. (5) defines the aberration $\Delta V_{i j}$ of each pixel curve $V_{i j}$ from 
the normal curve $\left\langle V_{i j}\right\rangle$ depending on pixel voltage $V_{i j}$. This relation is also linear (see Fig. 3b) and can be determined using the least-square method applied on at least two supporting points. These supporting points are infrared images taken from a blackbody covering the whole $F_{0} V_{\text {scene }}$ at different temperatures. Neither the absolute value of detected irradiance nor the absolute temperature of the blackbody is needed for the NUC.

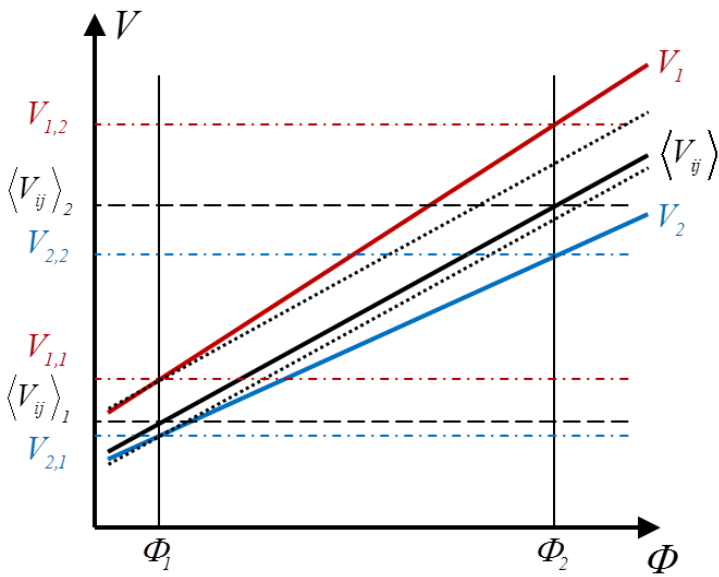

(a)

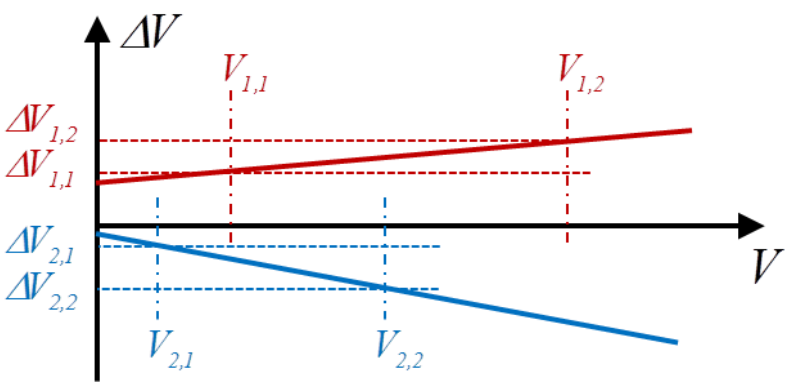

(b)

Fig 3. Linear non-uniformity correction of pixel response curves. (a) Relation between exchanged radiation and pixel responds, (b) pixel-specific aberration from normal curve.

According to Equ. (3), another influence on the amount of detected radiation originates from the camera interior. Due to its dependence on ambient temperature the two infrared images have to be taken at the same ambient temperature and within a steady-state temperature distribution inside the camera. The results of the linear regression are two pixel-specific parameter sets $a_{i j}$ and $b_{i j}$ correlating with the aberration of the pixel gains and offsets, respectively.

$$
\begin{gathered}
\Delta V_{i j}=V_{i j}-\left\langle V_{i j}\right\rangle=a_{i j} V_{i j}+b_{i j} \\
\left\langle V_{i j}\right\rangle=\left(1-a_{i j}\right) V_{i j}-b_{i j}
\end{gathered}
$$

These sets form the correction scheme of the two-point correction of the pixel signal voltage $V_{i j}$. After applying the NUC on the uncorrected infrared image the composition of radiation to be measured and disturbing radiation in Equ. (3) changes:

$$
V_{N U C}=\left\langle V_{i j}\right\rangle=\left\langle R_{V, i j} A_{i j}\right\rangle\left[\left\langle\omega_{O b j, i j}\right\rangle L\left(\vartheta_{O b j}\right)+\left\langle\int_{\omega_{C a m, i j}} L\left(\delta \vartheta_{C a m 0}, \omega\right) d \omega-M_{i j}\right\rangle\right]+\left\langle V_{O, i j}\right\rangle
$$

One may notice that the gain correction of the NUC not only equalizes the pixel-specific deviation of the responsivity $R_{V, i j}$ but also the pixel variation in reduced solid angle $\omega_{O b j, i j}$ related to the object. It should be recalled that the NUC is bound to the steady-state at one particular ambient temperature or - more accurately - one certain camera temperature distribution $\delta \vartheta_{\text {Cam } 0}$. This correction is essential for every infrared sensor array used for temperature distribution measurements because this step relates each pixel signal voltage to each other.

\section{NUC measurement results}

For our study we used two infrared cameras containing different microbolometer FPA sensors (ULIS, France), one with and another one without integrated TEC for temperature stabilization (table 1). The transmission of the raw (uncorrected) infrared images from the camera to a personal computer (PC) was implemented by a camera link interface. The data acquisition was performed by a self-written program. Matlab was used for digital signal processing operations. 
Table 1. Properties of used infrared cameras. (ULIS, France)

\begin{tabular}{|l|l|l|}
\hline Camera & $\mathbf{\# 1}$ & $\mathbf{\# 2}$ \\
\hline Sensor type & UL04171-011 & UL03162-028 \\
\hline TEC & integrated & w/o \\
\hline NETD & $<60 \mathrm{mK}(\mathrm{F} / 1,300 \mathrm{~K}, 60 \mathrm{~Hz})$ & $<100 \mathrm{mK}(\mathrm{F} / 1,300 \mathrm{~K}, 50 \mathrm{~Hz})$ \\
\hline Resolution & $640 \times 480$ & $384 \times 288$ \\
\hline Pixel pitch & $25 \mu \mathrm{m}$ & $25 \mu \mathrm{m}$ \\
\hline Uniformity (deviation) & $<1.5 \%$ & $<1.5 \%$ \\
\hline Power consumption & $<300 \mathrm{~mW}$ & $<100 \mathrm{~mW}$ \\
\hline f-number & 1.0 & 1.0 \\
\hline Focal length & $25 \mathrm{~mm}$ & $18 \mathrm{~mm}$ \\
\hline
\end{tabular}

Both infrared cameras were treated in the same way. During the calibration process the infrared cameras were placed inside a climate chamber adjusted at a chamber temperature of $20^{\circ} \mathrm{C}$. After one hour the cameras had reached the thermal steady-state. A water bath blackbody with a radiation area of $350 \mathrm{~mm} \times 350 \mathrm{~mm}$ was placed in front of a small opening inside one side of the climate chamber. The temperature of the blackbody was controlled between $10{ }^{\circ} \mathrm{C}$ and $40^{\circ} \mathrm{C}$ in steps of $5 \mathrm{~K}$. The waiting time between each blackbody temperature change amounted to 50 minutes. Figure 4 shows this calibration set-up.

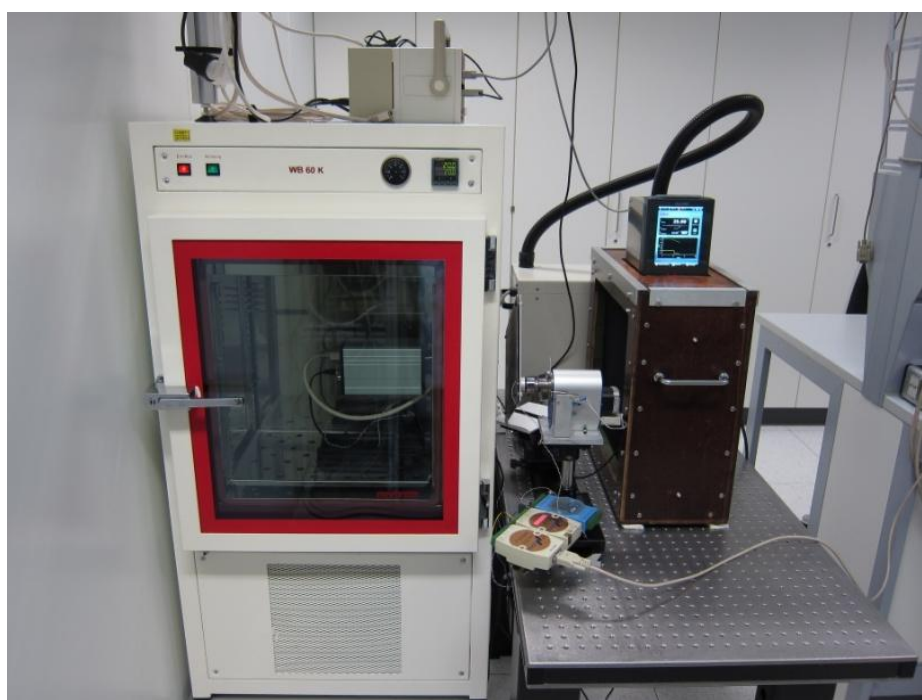

Fig. 4. Calibration set-up consisting of climate chamber with infrared camera and water bath blackbody.

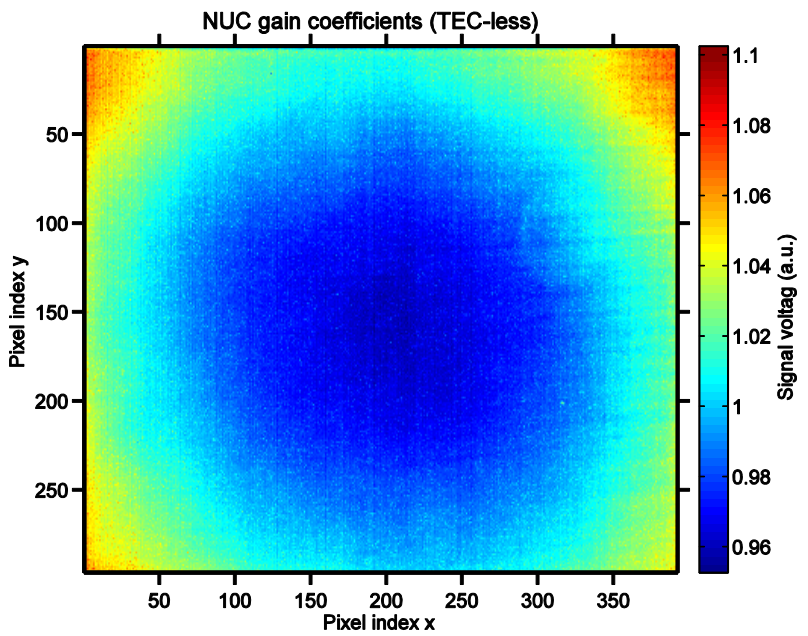

(a)

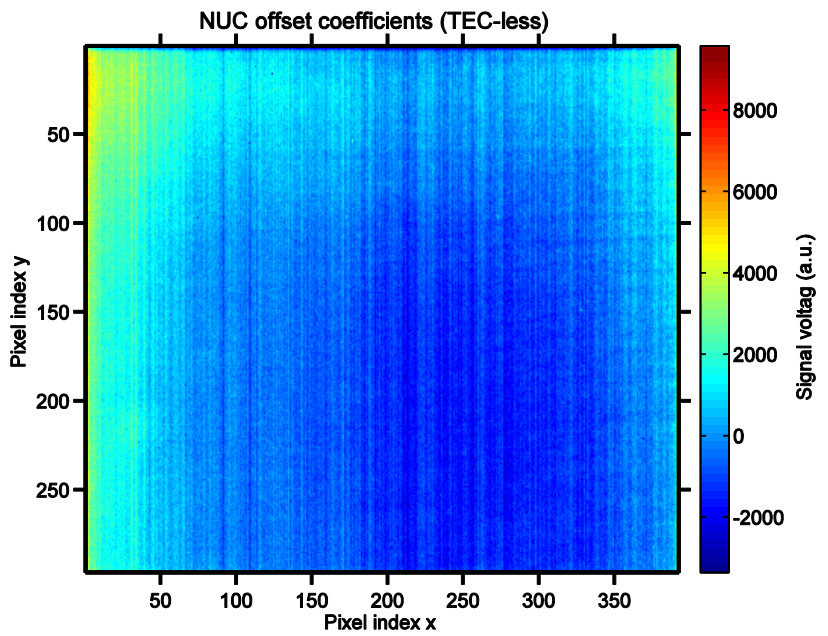

(b)

Fig 5. Exemplary distribution of NUC coefficients over all pixels of a FPA (IR camera \#2): (a) gain and (b) offset.

The NUC coefficient sets were determined based upon the infrared images at blackbody temperatures of $10^{\circ} \mathrm{C}$ and $40^{\circ} \mathrm{C}$ (Figure 5). Figure 6 shows the residual non-uniformity after NUC for all taken infrared images. One may notice 
that the resulting spatial standard deviation over the corrected infrared images is ca. 10 a.u. (arbitrary units), except at the two images on which the NUC is based. It can also be seen that the NUC procedure is independent of the blackbody temperature. The residual deviation is caused only by the temporal signal noise of each pixel.

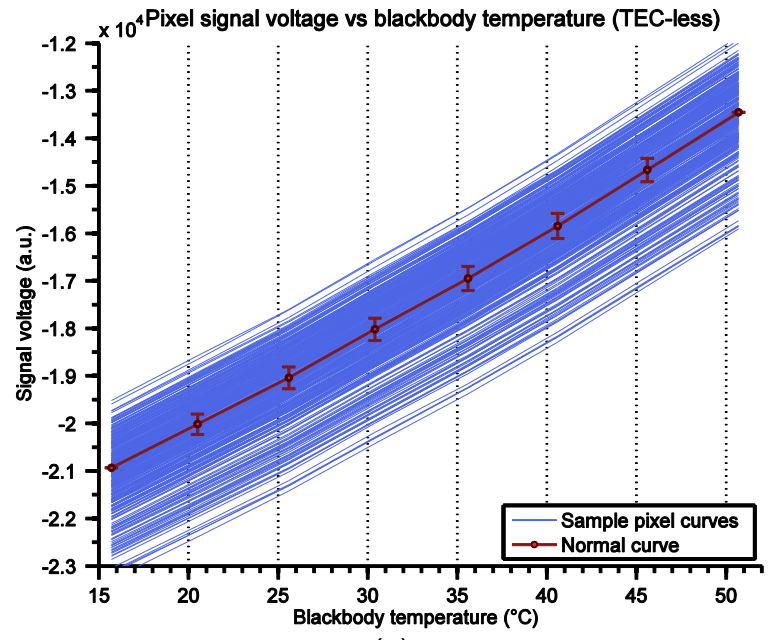

(a)

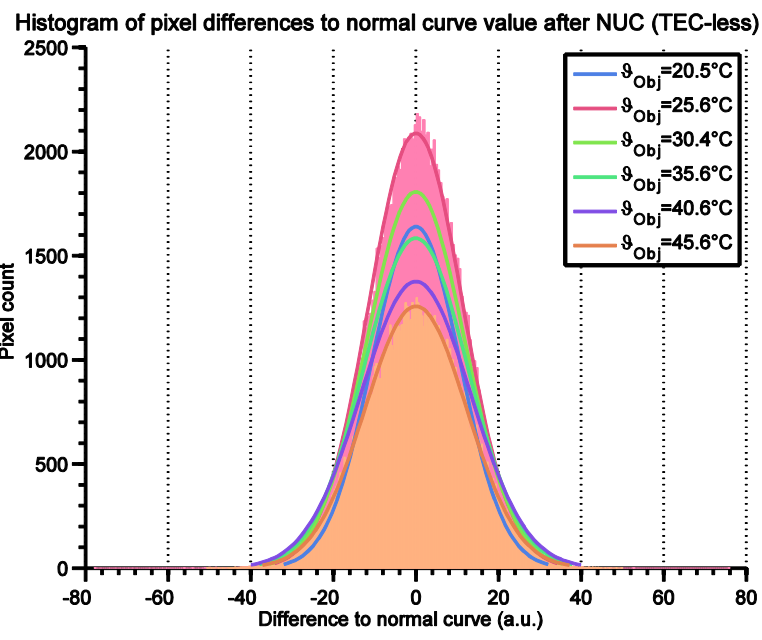

(b)

Fig 6. (a) Response curves (blue) of all FPA pixels of a particular line from IR camera \#2 before NUC. Red curve depicts the normal curve (indicated standard deviation values are amplified by 20 for better visualization), (b) standard deviation to normal curve as histograms. Lines show fitting curves assuming a normal distribution.

\section{Thermal drift}

As mentioned in Section 4 the applied non-uniformity correction assumes a constant ambient temperature and, in consequence, a constant temperature distribution $\delta \vartheta_{\text {Camo }}$ inside the camera housing. This condition cannot be ensured for most of the measurement scenarios. A variation in ambient temperature results in changes of the temperature distribution $\delta \vartheta_{\text {Cam }}$ inside the camera and its emitted radiation $\Delta L\left(\delta \vartheta_{\text {Cam }}, \omega\right)$ :

$$
V_{N U C, i j}=V_{N U C}+\left\langle R_{V, i j} A_{i j}\right\rangle \int_{\omega_{C a m, i j}} \alpha(\omega) \Delta L\left(\delta \vartheta_{C a m}, \omega\right) d \omega
$$

Figure 7 illustrates the impact of a change in ambient temperature of $10^{\circ} \mathrm{C}$. The observed scene has been kept constant. Both infrared images were taken with infrared camera \#1 using temperature stabilization after NUC; the first image at reference ambient temperature, the second after raised ambient temperature using the climate chamber. The camera temperature increases as a consequence also by about $10^{\circ} \mathrm{C}$. According to Equ. (8) the changes in the signal pixel voltages are non-uniform.

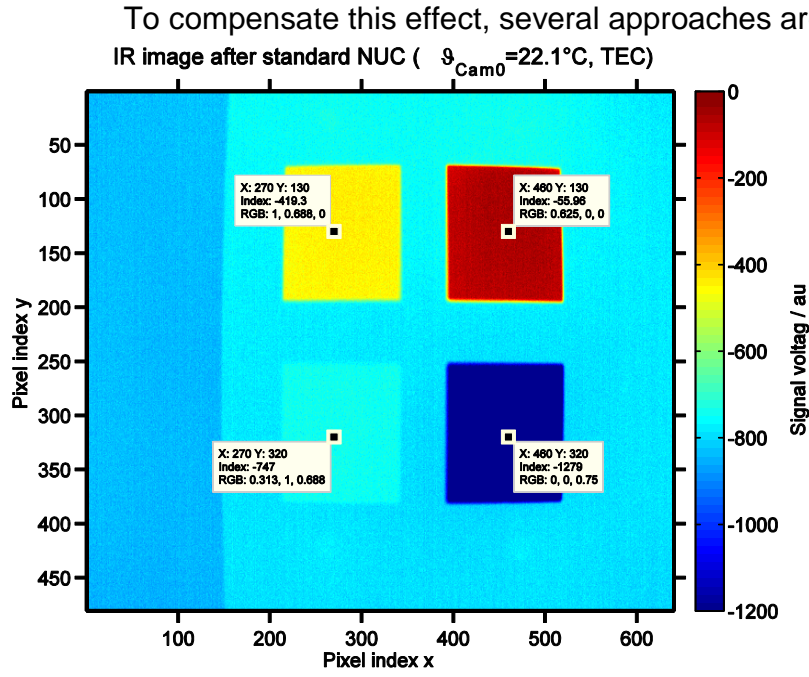

(a)

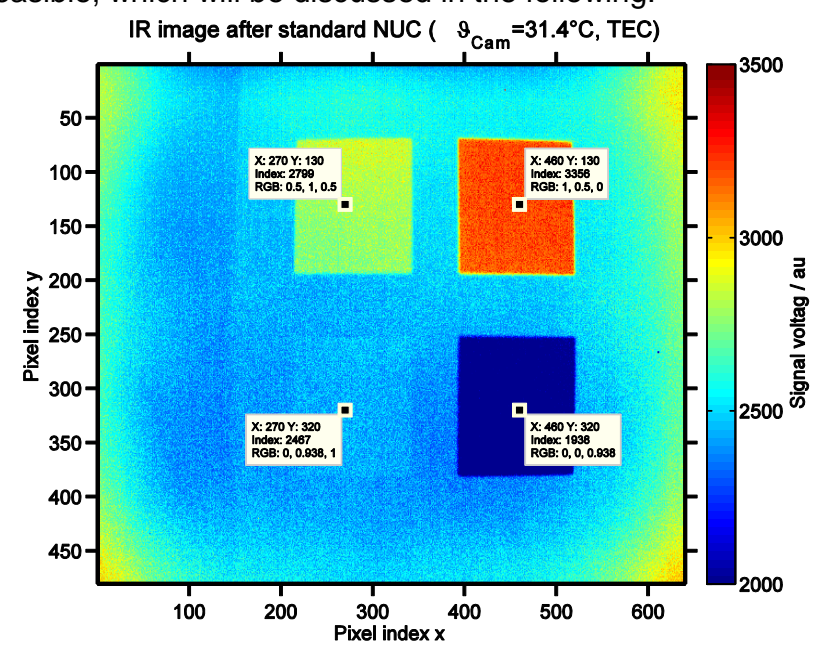

(b)

Fig 7. Impact of increasing camera temperature on infrared measurement. Infrared image after NUC at reference ambient temperature (a). NUC infrared image after raised ambient temperature (b). 


\subsection{Periodic recalibration using auxiliary components}

The simplest compensation approach is to measure the influence on the signal voltage due to the disturbing radiation flux directly by the same infrared FPA sensor. Since it is not possible to distinguish the origin of each part of irradiance a uniform radiation source, e.g. a blackbody, shutter etc., is needed as reference. After determining the correction values they are subtracted from the measurement signal. This is achieved by updating the offset voltage correction coefficients $b_{i j}$ from Equ. (6) periodically using a fix update interval or after a certain temperature change inside the camera. The offset coefficients are redefined each time. The corrected measurement signal jumps due to the time-discrete recalibration. A disadvantage of this method is that the simple correction procedure needs a more complex measurement set-up due to the additional mechanically moved parts.

\subsubsection{Blackbody}

The simplest way to redefine the offset voltage correction coefficients is to repeat one calibration step. The infrared camera is periodically faced at a blackbody at a certain constant temperature. In this case all influences of the camera housing and the lens system are taken into account what reduces the measurement uncertainty. The aberration of each pixel voltage from the mean signal voltage forms the new offset coefficients $b_{i j}$. The measured radiation from the blackbody yields a constant offset voltage which can be neglected since it is constant. The obvious disadvantage consists of the presence of a blackbody at any time of measurement. However, in practice this approach is adapted very often.

\subsubsection{Shutter}

It is very common to replace the blackbody by an internal optical shutter covering the whole camera field of view [1]. The shutter temperature and its radiant exitance depend on the camera temperature and are not constant compared to the blackbody temperature as in Section 5.1.1. The relation between the signal voltage $V_{\text {open }}$ with an opened and $V_{\text {closed }}$ for the closed shutter at various camera temperatures defines the shutter curve (Fig. 8). This correlation has to be determined for each infrared camera during the calibration process since it depends on the dimensions of the infrared camera, e.g. the distance between FPA and pupil and the aperture size. The linear gain of the shutter curve is the additional weight $c_{\text {open_closed }}$ which allows shifting the corrected signal voltage back into the desired voltage range:

$$
\left\langle V_{i j}\right\rangle=\left[\left(1-a_{i j}\right) V_{i j}-b_{\text {shutter }, i j}\left(\delta \vartheta_{\text {Cam }}\right)\right] \cdot c_{\text {open_closed }}
$$

Combining all mobile parts into one single camera housing makes this approach very useful in practice. The shutter can be placed close to the FPA or in front of the first lens. This position influences the measurement uncertainty. Thermal influences of the lens system are not considered. However, the disadvantage of an interrupted measurement remains. The construction and the miniaturization of the infrared camera are limited due to the shutter size.

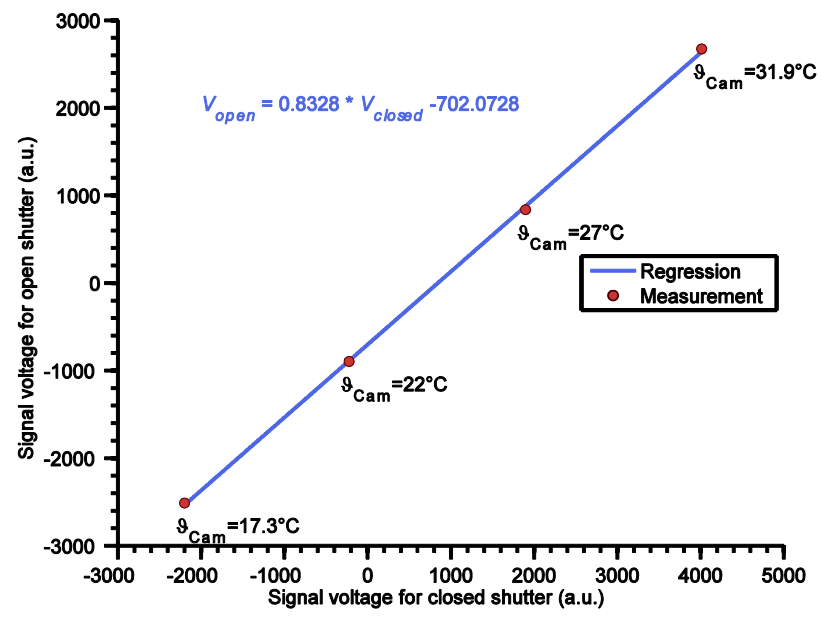

Fig 8. Measurement of the shutter curve at several ambient temperatures and camera temperatures respectively and regression curve. The gain value of the regression curve corresponds to $c_{\text {open_closed }}$. 


\subsubsection{IR filter and IR emitter}

A different approach is described in [5] using an infrared filter instead of the shutter. The transmittance $\tau_{\text {filter }}$ of the filter has to be uniform and well known. It is introduced periodically in front of the infrared camera covering the entire field of view. Using two subsequent infrared frames - one with and another without infrared filter - the offset voltage correction coefficients are determined. It is assumed that changes in the observed scene can be neglected due to the short measurement interval. It was also shown that the thermal drift can be compensated with the knowledge of the filter's optical properties and position.

Due to the semitransparent infrared filter a continuous measurement can be performed. The uncertainty of the infrared filter properties and its uniformity are new sources for the measurement uncertainty. The complexity of the measurement set-up is not reduced compared to the approaches mentioned from Sections 5.1.1 and 5.1.2.

\subsection{Continuous recalibration}

All previously described approaches use additional components for recalibration which conflicts with the aim of a reduced infrared measurement set-up comprising only the sensor array, the lens system and the data processing unit. This might be achieved by taking advantage of the spatial and temporal behavior of the pixel signal voltages or other parameters being in close relation to the thermal drift.

\subsubsection{Scene-based NUC}

An overview on basic methods of scene-based correction is given in [6] and [7]. These approaches are based on an arbitrary moving scene. Averaged over a long period of time it can be seen as uniform radiation source. Due to this uniform background each single sensor element should show the same temporal mean signal voltage. The offset voltage is corrected by subtracting the difference of the previous pixel signal voltage to the mean background voltage from the current pixel signal voltage. The weights of the applied moving average algorithm should be optimized for the particular measurement set-up and application because of their effects on the resulting measurement uncertainty.

An improved scene-based algorithm sets up a neuronal network including the local neighborhood of each pixel. In a hidden layer these signal values are weighted and summed forming a spatial-temporal filter. The results are used to predict the desired output value from each new input. This approach requires a constantly motion of the scene. The performance can be increased using different neuronal networks with a higher complexity.

The main disadvantage of scene-based techniques is the requirement of continuous moving of the scene in relation to the sensor array. That is the reason why the achieved measurement uncertainty is always influenced by the background of the observed scene which may lead to artifacts in the corrected infrared image. These approaches cannot be applied to immobile scene scenarios.

\subsubsection{Indirect correction using sensor temperature}

In [8] a completely different approach is used to compensate the effects of the thermal drift. Here, the infrared camera contains a TEC-less FPA sensor which provides the internal sensor temperature as an additional parameter at any time during the measurement. The sensor temperature is in close correlation to the temperature distribution conditions inside the camera due to heat conduction and convection inside the camera housing. Each pixel of the sensor array is seen as an individual sensor element and, therefore, corrected independently from the other pixels. They use a climate chamber to increase the ambient temperature from $5^{\circ} \mathrm{C}$ up to $65^{\circ} \mathrm{C}$ in steps of $5 \mathrm{~K}$ during the calibration process. After a settling time of one hour the pixel voltages are determined. The relation between the ambient temperature as well as the inside temperature distribution and the detected radiant flux is nonlinear, but it can be approximated by a polynomial of second degree if the ambient temperature stays within the boundaries of the calibration process. With these correction functions and the sensor temperature input it is possible to calculate the aberration of each pixel signal voltage due to changes in ambient temperature.

This approach is based on an extended calibration procedure which demands special equipment, e.g. a climate chamber. Due to the fact that the correction parameters for each pixel have to be determined individually, the calculation time is high and increases dramatically for sensor arrays with higher resolution. Another disadvantage is the increasing measurement uncertainty if the ambient temperature exceeds the thermal boundaries predetermined by the calibration process. This correction is based only on the steady-state behavior of the inside camera temperature distribution. This results in a long settling time after an abrupt change in ambient temperature until the correction has reached an acceptable measurement uncertainty.

\subsubsection{Proposed approach}

We suggest an approach based on [8]. First, we follow the method described in [4] in order to compensate the effects of a TEC-less operating infrared FPA sensor. If the infrared camera utilizes a temperature-stabilized sensor array this step could be skipped. The infrared camera is exposed to a changing ambient temperature using a climate chamber. As mentioned in Section 5.2.2 these temperature changes affect also the sensor temperature. The effects on the sensor 
responsivity and the fix-pattern offset values due to changes in sensor temperature are considered. It is not possible to distinguish between the influences derived from changes in irradiation from the camera housing due to the changing camera temperature and the changes in fix-pattern offset values due to changes of the sensor temperature. In [4] each calibration measurement is done after applying a shutter-based recalibration. The internal shutter has to be replaced by auxiliary components during the calibration procedure. Once the correction parameters are determined these auxiliary components are not required for the measurements. Second, we use another parameter than the sensor temperature which shows a closer correlation between the temperature distribution inside the camera housing and the changes in detected irradiance due to changes in the temperature distribution. Assuming that those changes of the inside camera temperature conditions are uniform it is possible to reduce the amount of corrections curves mentioned in Section 5.2.2. Figure 9 illustrates the work flow of the proposed calibration procedure of a microbolometer-based infrared camera.

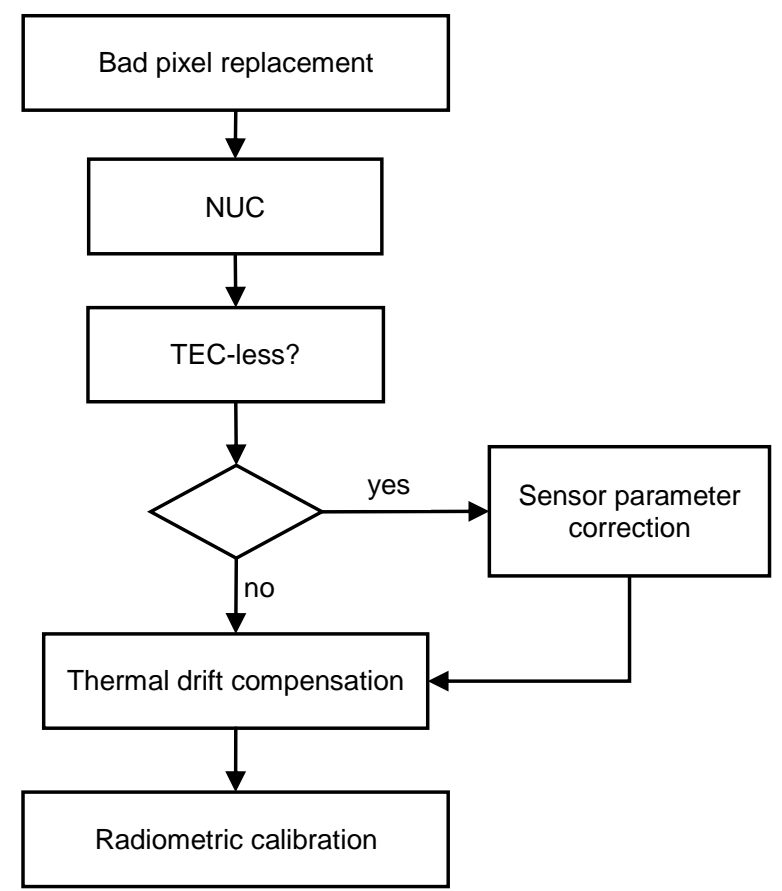

Fig 9. Workflow diagram of the proposed calibration procedure of an infrared camera [4].

\section{Conclusion}

None of the existing methods meet completely the demands for the desired aim of a minimalistic infrared camera which would open the field for new infrared measurement applications. The standard approach with shutter or other auxiliary components is not suitable because of the requirements for small size and miniaturization. Since a number of these new applications are based on a fix mounted infrared camera observing a stationary scene, scene-based methods for thermal drift compensation cannot be applied either because of their strict requirement of a continuously moving scene background. Determining the disturbing radiation flux indirectly seems to be the most promising approach which meets all demands.

\section{Acknowledgements}

This work is financially supported by the Deutsche Forschungsgemeinschaft Germany under contract GE779/26-1.

\section{REFERENCES}

[1] Budzier, H., Gerlach, G., "Thermal Infrared Sensors”. Wiley, Chichester, 2011.

[2] http://www.ulis-ir.com/uploads/Products/BrochureMicro80p-(Web).pdf

[3] Tempelhahn, A., Budzier. H., Krause. V., Gerlach, G., "Modeling signal-determining radiation components of microbolometer-based infrared measurement systems". AMA Conferences 2013, Nuremberg, 14.-16.5.2013, Proceedings IRS22013, AMA Services 2013, 100-104.

[4] Budzier, H., "Radiometrische Kalibrierung ungekühlter Infrarot-Kameras". TUDpress, Dresden, 2014.

[5] Olbrycht, R., Wiecek, B., De Mey, G., "Thermal drift compensation method for microbolometer thermal cameras". Applied Optics 51 (2012) 11, 1788-1794. 
[6] Narendra, P. M., Foss, N. A., "Shutterless fixed pattern noise correction for infrared imaging arrays". Technical Issues in Focal Plane Development, Proceedings of SPIE, Vol. 282, 44-51, 1981.

[7] Scribner, D. A., Sarkady, K. A., Caulfield, J. T., Kruer, M. R., Katz, G., Gridley, C. J., "Nonuniformity correction for staring IR focal plane arrays using scene-based techniques". Infrared Detectors and Focal Plane Arrays, Proceedings of SPIE, Vol. 1308, 224-233, 1990.

[8] Bieszczad, G., Orzanowski, T., Kastek, M., "Method of detectors offset correction in thermovision camera with uncooled microbolometric focal plane array". Electro-Optical and Infrared Systems, Proceedings of SPIE, Vol. 7481, 748100-1-8, 2009. 